\title{
A High Precision Direct Integration Scheme Based on Variational Principle and Its Applications
}

\author{
Shuai Zhu ${ }^{1}$, Gang Zhou ${ }^{2}$, Xiaomei Liu ${ }^{3}$ and Shilie Weng ${ }^{1}$ \\ ${ }^{1}$ Shanghai Jiao Tong University, School of Mechanical Engineering, 200240 Shanghai, China \\ ${ }^{2}$ Shanghai Jiao Tong University, Department of Mathematics, 200240 Shanghai, China \\ ${ }^{3}$ Shanghai Second Polytechnic University, Department of Mathematics, 201209 Shanghai, China
}

\begin{abstract}
Dynamics response of systems to impact or loading may be effectively treated by direct integration. However, it is often difficult to select the time-step of integration properly, especially in the case which the system is badly stiff. High Precision Direct integration based on variational principle is given (HP D-VP) for homogeneous systems and HHPD-VP method for the nonhomogeneous systems are given. This method not only takes the advantage of variational principle formula, which is much precise and is stiff A-stable, but also can avoid the truncation error of the computer. For the large systems, especially, the systems with different frequency or the stiff systems, our methods are stable, accurate and efficient. Numerical experiments show the convergence order of the scheme derived from the variational principle, and is much precise and is effective in engineering.
\end{abstract}

\section{Introduction}

Dynamic response is important for engineering. Such responses are analysed by means of direct integration methods, such as the Newmark- $\alpha$, Wils on $-\theta$ or Houbolt schemes. When using such methods, the time-step size must be carefully selected, especially for some stiff equations. As a result, these direct integration schemes require a very small time-step and are generally timeconsuming and costly. Zhong [1] proposed High Precise Direct (HPD) method for homogeneous linear timeinvariant dynamic systems. HPD is more accurate than traditional R-K method and Newmark method, which is very valuable in engineering applications. For nonhomogeneous systems, Zhong [2] also established HPD$\mathrm{L}$ algorithm, in which the non-homogeneous term is simulated by piecewise linearization within a time step.

Meanwhile, Lin [3] HPD-F method, which is based on Fourier expansion, is also proposed. However, HPD-L and HPD-F have to compute the inverse matrix. Zhou [4] [5] and $\mathrm{Gu}$ [6] proposed another improved HPD method independently, which transformed the non-homogeneous term into homogeneous by expanding dimensions. Shi [7] and $\mathrm{Fu}[8]$ established HHPD-L and HHPD-C, which are based on Legendre and Chebyshev Serials, respectively.

From the viewpoint of computation, finite element (FE) method is also a useful tool to discretize the time domain of structural dynamic problems. Early development on variational for time integration have been introduced by Argyris and Scharpf [9]. Another approach to discrete time is discontinuous Galerkin method, which was first proposed by Reed and Hill [10], due to the flexible shape function, DG has been widely used in many areas of computation. Cockburn [11] gives LDG method, which is time-stepping. The time discontinuous Galerkin method leads to stable, higherorder accurate finite element methods, which is shown in Delfour [12]. Gottlieb [13] gives the unified discontinuous Galerkin framework for time integration, which show some special discontinuous Galerkin method equal to finite difference methods. [14] presented an improved predictor or multi corrector solution algorithm based on the concept of time discontinuous Galerkin (TDG) FE method to compute the dynamic response of linear structures using the Gauss-Seidel method in an iterative way.

We propose HPD-VP method for the homogeneous systems and HHPD-VP method for the nonhomogeneous systems, which base on the variant principle, and can lead the stable schemes. Firstly, HPD-VP and HHPD-VP are highly precise, compared to the traditional method, such Newmark- $\alpha$, Wils on- $\theta$, Runge-Kutta and so on. Secondly, HPD-VP and HHPD-VP are stiff A-stable, for the large systems especially, the stiff equations, our methods are reliable, and can simulate the mix frequency systems with large time step.

\section{A Novel Variational Principle Approach}

A novel variational principle approach for solving the following initial value problem of linear differential equation. 


$$
\left\{\begin{array}{c}
\frac{d u}{d t}=A u+f(t), \quad 0<t<T \\
u(0)=u_{0}
\end{array}\right.
$$

The Eq. (1) can be technically (see [4] [7] [8] [6]) transformed into

$$
\left\{\begin{array}{c}
\frac{d \tilde{u}}{d t}=\widetilde{A} \widetilde{\boldsymbol{u}}, \quad 0<t<T \\
\widetilde{\boldsymbol{u}}(\mathbf{0})=\widetilde{\boldsymbol{u}}_{0}
\end{array}\right.
$$

which is the homogeneous ODE.

For Eq. (2), consider a partition of a closed interval $[0, \mathrm{~T}]: 0=t_{0}<t_{1}<\cdots<t_{N-1}<t_{N}=\mathrm{T}$. The interval $I_{n}:=\left[t_{n}, t_{n+1}\right]$ and $h_{n}$ its length $h_{n}:=t_{n+1}-t_{n}$ Establish the variational principle method for the Eq. (2). To describe the variational principle method, consider the following notation. The space of polyno mials of degree $q$ or less on the interval $I_{n}$.

$$
\mathrm{P}_{q}\left\{I_{n}\right\}=\left\{v: v(t)=\sum_{i=0}^{q} v_{i} t^{i}\right\}
$$

and the space contains the piecewise polynomials to be

$$
\mathrm{U}=\left\{U:\left.U\right|_{t_{n}} \in \mathrm{P}_{q}\left\{I_{n}\right\}\right\}
$$

In the variational principle method, the trail function is continuous within each time interval $I_{n}$. At each node $t_{n}$, the limiting values of numerical approximations from the left and the right are usually different.

The jump,

$$
\begin{aligned}
& \boldsymbol{u}_{n}^{+}=\lim _{s \rightarrow 0^{+}} \boldsymbol{u}\left(t_{n}+s\right) \\
& \boldsymbol{u}_{n}^{-}=\lim _{s \rightarrow 0^{-}} \boldsymbol{u}\left(t_{n}+s\right)
\end{aligned}
$$

$$
\left[\boldsymbol{u}_{n}\right]=\boldsymbol{u}_{n}^{+}-\boldsymbol{u}_{n}^{-}
$$

Then, Eq. (2) can be expressed as follows,

Find $\boldsymbol{u} \in U$

$$
\sum_{n=1}^{N}\left[\int_{I_{n}}\left(\frac{\boldsymbol{d} \widetilde{\boldsymbol{u}}}{\boldsymbol{d} \boldsymbol{v}} \boldsymbol{v}-\widetilde{\boldsymbol{A}} \widetilde{\boldsymbol{u}} \boldsymbol{v}\right) d t+\left[\widetilde{\boldsymbol{u}}_{n}\right] \boldsymbol{v}_{n}^{+}\right]=0
$$

Since $\boldsymbol{v}$ is varies independently on each interval $I_{n}$, this can be written as

$$
\int_{I_{n}}\left(\frac{d \widetilde{u}}{d t} \boldsymbol{v}-\widetilde{\boldsymbol{A}} \widetilde{\boldsymbol{u}} \boldsymbol{v}\right) d t+\left[\widetilde{\boldsymbol{u}}_{n}\right] \boldsymbol{v}_{n}^{+}=0
$$

For $q=1$, we take shape function $\phi_{1}=(1-\xi) / 2$, $\phi_{2}=(1+\xi) / 2$, and test function $\phi_{i}, i=1,2$, where the shape function and test function are in the same function space, namely.

$$
\tilde{\boldsymbol{u}}=\phi_{1} \tilde{\boldsymbol{u}}_{1}+\phi_{2} \tilde{\boldsymbol{u}}_{2}
$$

and insert the shape functions and test functions into Eq. (4).

Thus, we have

$$
\widetilde{\boldsymbol{u}}_{n}^{-}=\frac{\left(I+\frac{\widetilde{A} h}{3}\right)}{\left(\frac{\tilde{A}^{2} h^{2}}{6}-\frac{2 \widetilde{A} h}{3}+I\right)} \widetilde{\boldsymbol{u}}_{n-1}^{-}
$$

Denote

$$
\mathbf{W}=\frac{\left(I+\frac{\nexists \mathcal{A} h}{3}\right)}{\left(\frac{A^{2} h^{2}}{6}-\frac{2 A h}{3}+I\right)}
$$

W is the sub-diagonal Páde approximation $R_{1,2}(\widetilde{A} h)$ with with error estimate

$$
\left|\mathbf{W}(\widetilde{A} h)-e^{(\widetilde{A} h)}\right|=\boldsymbol{O}\left(h^{\mathbf{1}+2+1}\right)=\boldsymbol{O}\left(h^{\mathbf{4}}\right)
$$

Since

$$
\mathbf{W}(\widetilde{A} h) \rightarrow 0 \text { as } \operatorname{Re}(\widetilde{A} h) \rightarrow \infty
$$

So this scheme is stiff A-stable.

\section{The Higher Precise Integral Based on Variant Principle}

For the first interval length $h=\left[t_{1}-t_{0}\right]$, we can equally divide it into $2^{N}$, so the each length of subdivision is $\tau=h / 2^{N}$, and N is the parameter of HPD-VP method. The following shows how to computer $\mathbf{W}(\widetilde{A} h)$.

$$
\mathbf{W}(\widetilde{A} h)=\frac{\left(I+\frac{\widetilde{A} h}{3}\right)}{\left(\frac{\widetilde{A}^{2} h^{2}}{6}-\frac{2 \widetilde{A} h}{3}+I\right)}=\left[\frac{\left(I+\frac{\widetilde{A} \tau}{3}\right)}{\left(\frac{\widetilde{A}^{2} \tau^{2}}{6}-\frac{2 \widetilde{A} \tau}{3}+I\right)}\right]^{2^{N}}
$$

Denote

$$
\begin{aligned}
& \mathbf{W}(\widetilde{\boldsymbol{A}} h)=[\mathbf{W}(\widetilde{\boldsymbol{A}} \tau)]^{2^{N}}=:\left[\mathbf{I}+\mathbf{T}_{\boldsymbol{\alpha}, \mathbf{0}}\right]^{2^{N}} \\
& \text { Since } \\
& {\left[\mathbf{I}+\mathbf{T}_{\alpha, 0}\right]^{2}=I+2 \mathrm{~T}_{\alpha, 0}+\mathrm{T}_{\alpha, 0} \mathrm{~T}_{\alpha, 0}=: I+\mathrm{T}_{\alpha, 1}} \\
& {\left[\mathbf{I}+\mathbf{T}_{\alpha, 1}\right]^{2}=I+2 \mathbf{T}_{\alpha, 1}+\mathbf{T}_{\alpha, 1} \mathbf{T}_{\alpha, 1}=: I+\mathbf{T}_{\alpha, 2}} \\
& {\left[\mathbf{I}+\mathbf{T}_{\alpha, N-1}\right]^{2}=I+2 \mathrm{~T}_{\alpha, N-1}+\mathrm{T}_{\alpha, N-1} \mathrm{~T}_{\alpha, N-1}=: I+\mathrm{T}_{\alpha, N}}
\end{aligned}
$$
(6)

\section{Convergence of HPD-VP}

Assume the error of Eq. (6) is $\epsilon$, then, $\epsilon$ is subject to

$$
\|\epsilon\|_{2}=o(\tau d \tau)
$$

PROOF

For the initial value problem Eq. (2), it has the precise solution

$$
\begin{aligned}
{[T(h)] } & =\exp ([\widetilde{\boldsymbol{A}}] \times h)=\exp \left([\widetilde{\boldsymbol{A}}] 2^{N} \tau\right) \\
& =I+2^{N} \tau[\widetilde{\boldsymbol{A}}]+\frac{1}{2}\left(2^{N} \tau\right)^{2}[\widetilde{\boldsymbol{A}}]^{2}+\cdots
\end{aligned}
$$

and the HPD-VP method has the approximation,

$$
\begin{gathered}
{[I+\tau \widetilde{\boldsymbol{A}}]^{2^{N}}=I+C_{2^{N}}^{1} \tau \widetilde{\boldsymbol{A}}+C_{2^{N}}^{2} \tau^{2} \widetilde{\boldsymbol{A}}^{2}+\cdots C_{2^{N} \tau^{2^{N}} \widetilde{\boldsymbol{A}}^{2^{N}}}} \\
\quad=I+2^{N} \tau \widetilde{\boldsymbol{A}}+\frac{1}{2} 2^{N}\left(2^{N}-1\right) \tau^{2} \widetilde{\boldsymbol{A}}^{2}+\cdots+\tau^{2^{N}} \widetilde{\boldsymbol{A}}^{2^{N}}
\end{gathered}
$$

where

$$
C_{m}^{n}=\frac{n !}{m !(n-m) !}
$$

Compared to Eq. (8) and Eq. (9), the error $\epsilon$ should be

$$
\|\epsilon\|_{2}=o(\tau d \tau)
$$

\section{Numerical Experiments}

\subsection{The systems with different frequencies}

For the multi-frequency systems, the canonical equations are stiff equation. [15] shows courant number should satisfy $h w \approx 1$ where $h$ is the length of time step, and $w$ is the frequency of the systems, which means for the high-frequency system, the traditional methods have to choose the tiny time step $h$, vice versa.

Consider the Hamiltonian system with different frequency signals. 
The canonical equations

$$
\begin{aligned}
H\left(p_{1}, q_{1}, p_{2}, q_{2}\right) & \\
& =\frac{1}{2}\left(50 p_{1}^{2}+\frac{1}{50} p_{2}^{2}+200 q_{1}^{2}\right. \\
& \left.+\frac{4}{50} q_{2}^{2}\right)
\end{aligned}
$$

$$
\left\{\begin{array}{c}
p_{1}^{\prime}=-200 q_{1} \\
p_{2}^{\prime}=-4 / 50 q_{2} \\
q_{1}^{\prime}=50 p_{1} \\
q_{2}^{\prime}=1 / 50 p_{2}
\end{array}\right.
$$

Initial conditions

$$
p_{1}(0)=2, p_{2}(0)=2, q_{1}(0)=0, q_{2}(0)=0 .
$$

Exact solution

$$
\begin{gathered}
p_{1}=2 \cos (100 t), q_{1}=\sin (100 t) \\
p_{2}=2 \cos \left(\frac{t}{25}\right), q_{2}=\sin \left(\frac{t}{25}\right)
\end{gathered}
$$

We solve the Hamiltonian system with HPD-VP and some existing methods, such as FSJS3 [16], RKN [17], SPRKN [18] and time finite element method [19].

Table 1. Formatting sections, subsections and subsubsections.

\begin{tabular}{|c|c|c|c|}
\hline Method & $\begin{array}{c}\text { HPD-VP } \\
(h=0.2)\end{array}$ & $\begin{array}{c}\text { TFE2[19] } \\
(h=0.0001)\end{array}$ & $\begin{array}{c}\text { FSJS3[16] } \\
(h=0.0001)\end{array}$ \\
\hline$p_{1}$ & $1.6135 \mathrm{e}-11$ & $1.6666 \mathrm{e}-6$ & $1.7708 \mathrm{e}-7$. \\
\hline$q_{1}$ & $8.0675 \mathrm{e}-12$ & $8.3329 \mathrm{e}-7$ & 0.2672 \\
\hline$p_{2}$ & $1.9429 \mathrm{e}-13$ & $1.194 \mathrm{e}-10$ & $2.1902 \mathrm{e}-13$ \\
\hline$q_{2}$ & $1.0425 \mathrm{e}-13$ & $6.1553 \mathrm{e}-11$ & $1.0828 \mathrm{e}-6$ \\
\hline Method & $\begin{array}{c}\text { HPD-VP } \\
(h=0.2)\end{array}$ & $\begin{array}{c}\text { SPRKN }[18] \\
(h=0.001)\end{array}$ & $\begin{array}{c}\text { RKN }[17] \\
(h=1 \mathrm{e}-6)\end{array}$ \\
\hline$p_{1}$ & $1.6135 \mathrm{e}-11$ & $1.007 \mathrm{e}-5$ & 0.8881. \\
\hline$q_{1}$ & $8.0675 \mathrm{e}-12$ & 0.23532 & 0.4441 \\
\hline$p_{2}$ & $1.9429 \mathrm{e}-13$ & $7.267 \mathrm{e}-14$ & 3.9573 \\
\hline$q_{2}$ & $1.0425 \mathrm{e}-13$ & $5.1605 \mathrm{e}-9$ & 1.9786 \\
\hline
\end{tabular}

The data in Tab. 1 are the the maximum of absolute errors with in $t \in[0 s, 600 s]$. As shown in Tab. 1 HPD-VP method can simulate the high and low frequency with the big time step. We show the error at different time, with figures as follows. As shown in Fig. 1, HPD-VP is accurate at different time with the relative large time step, however, when the step is larger, SPRKN, FSFS and TFE method are not stable, and lead to very large errors.
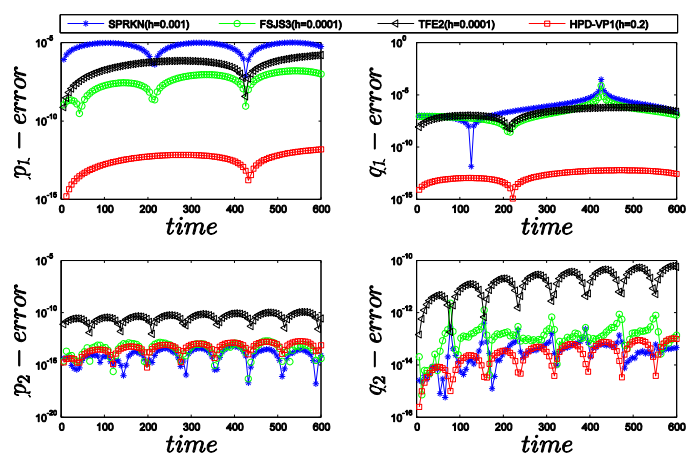

Figure 1. The error at different time by different method with different time step
We consider the solution of the 3 degree-of-freedom spring system [20]. For simplicity, all the three masses are assumed to be unity. The stiffness of the left spring is assumed to be $10^{7}$, while the stiffness of the right spring is unity. In addition, the displacement of the left point mass is set to be $\sin 2 t$, while the displacements of the other two masses are initially equal to zero. Hence, the equilibrium equations of this spring-mass system are given by

$$
\left(\begin{array}{l}
\ddot{u}_{1} \\
\ddot{u}_{2} \\
\ddot{u}_{3}
\end{array}\right)+\left(\begin{array}{ccc}
10^{7} & -10^{7} & 0 \\
-10^{7} & 10^{7}+1 & -1 \\
0 & -1 & 1
\end{array}\right)\left(\begin{array}{l}
u_{1} \\
u_{2} \\
u_{3}
\end{array}\right)=\left(\begin{array}{l}
r_{1} \\
0 \\
0
\end{array}\right)
$$

Where $r_{1}$ is the reaction force at the left point mass. The 3 degree-of-freedom spring systemshown in Fig. 2.

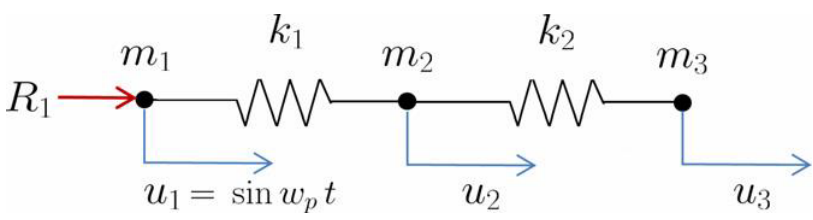

Figure 2. Model problem of three degrees of freedom spring system

Obviously, Eq. (10) is a stiff system. Since the displacement of the left node (i.e. $u_{1}$ ) is known, Eq. (10) can be rewritten as

$$
\left(\begin{array}{l}
\ddot{u}_{2} \\
\ddot{u}_{3}
\end{array}\right)+\left(\begin{array}{cc}
10^{7}+1 & -1 \\
-1 & 1
\end{array}\right)\left(\begin{array}{l}
u_{2} \\
u_{3}
\end{array}\right)=\left(\begin{array}{c}
10^{7} \sin 2 t \\
0
\end{array}\right)
$$
Let

with the initial values $u_{2}(0)=0$ and $u_{3}(0)=0$.

$$
\begin{gathered}
p=\sin 2 t \\
v_{2}=\dot{u}_{2} \\
v_{3}=\dot{u}_{3} \\
q=\dot{p}
\end{gathered}
$$

Then rewrite Eq. (11) by expanding dimensions as

$$
\begin{aligned}
& \left(\begin{array}{c}
u_{2} \\
u_{3} \\
p \\
v_{2} \\
v_{3} \\
q
\end{array}\right)= \\
& -\left(\begin{array}{cccccc}
0 & 0 & 0 & 1 & 0 & 0 \\
0 & 0 & 0 & 0 & 1 & 0 \\
0 & 0 & 0 & 0 & 0 & 1 \\
10^{7}+1 & -1 & -10^{7} & 0 & 0 & 0 \\
-1 & 1 & 0 & 0 & 0 & 0 \\
0 & 0 & 0 & 0 & 0 & 4
\end{array}\right)\left(\begin{array}{c}
u_{2} \\
u_{3} \\
p \\
v_{2} \\
v_{3} \\
q
\end{array}\right) \\
& \text { with the initial values } \\
& u_{2}(0)=0, u_{3}(0)=0, p(0)=0 \\
& v_{2}(0)=2, v_{3}(0)=0, q(0)=2,
\end{aligned}
$$

We solve Eq. (11) by using Euler time integration method, Newmark $-\alpha$ [21], Wilson- $\beta$ [22] with time step $\mathrm{h}=0.0005$, and HPD-VP method with time step $\mathrm{h}=0.5$. Fig. 3, Fig. 4 are the displacement response and Fig. 5, Fig. 6 are the velocity response. The exact solutions are obtained by the mode superposition method. We can get the conclusion that HHPD-VP method can highly increase the accuracy with big time step.

\subsection{Spring-mass system}




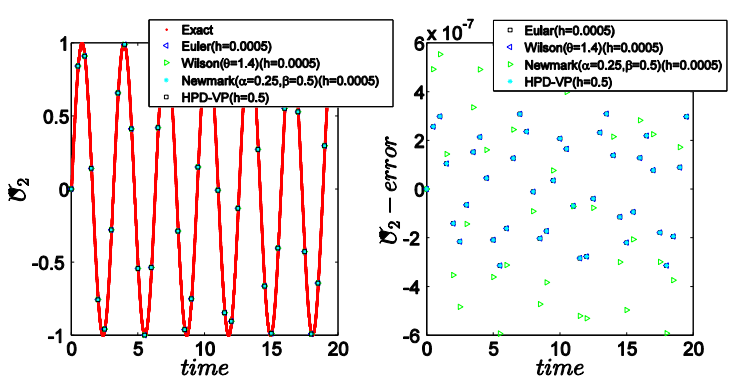

Figure 3. Displacement(right) and displacement error(left) of node 2 for various methods different time step

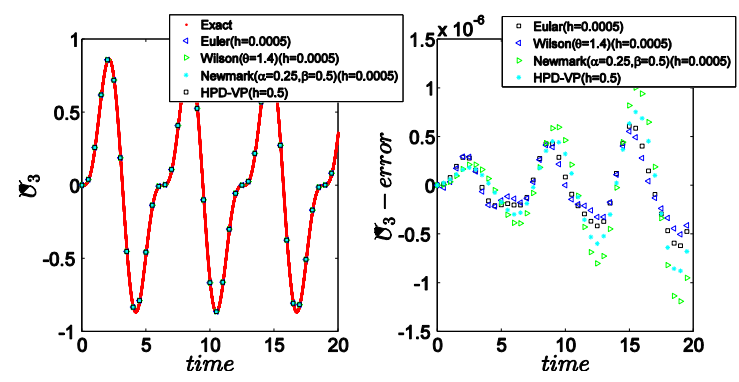

Figure 4. Displacement(right) and displacement error(left) of node 3 for various methods different time step

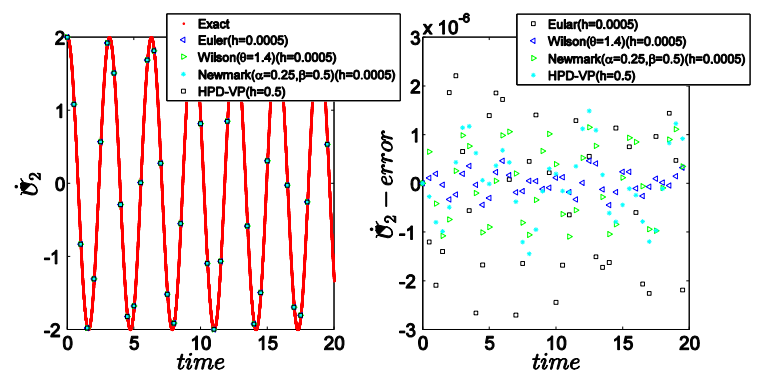

Figure 5. Velocity(right) and velocity error(left) of node 2 for various methods.

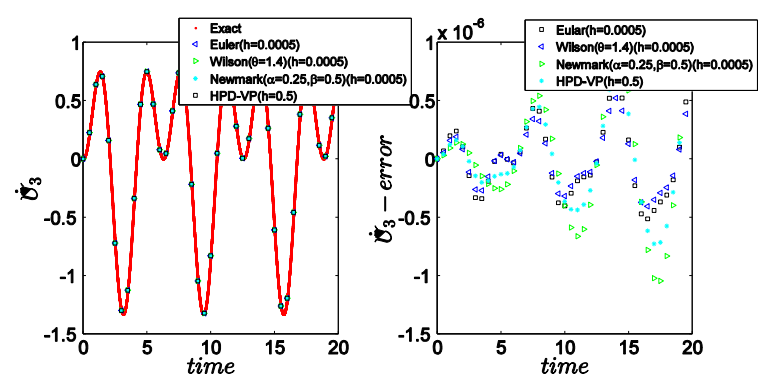

Figure 6. Velocity(right) and velocity error(left) of node 3 for various methods.

\section{Conclusion}

HPD-VP and HHPD-VP methods base on the variant principle, which are stiff A-stable methods, for large systems, our methods are stable, efficient and accurate. Especially for the systems with mix-frequency or the stiff system, traditional methods cannot simulate both high and low frequency with large time step, our method can solve this problem. HHPD-VP method avoid the computation of the inverse of matrix. For the dynamic systems, our method are reliable. The numerical experiments testify the results.

\section{References}

1. Zhong Wanxie. On precise time-integration method for structural dynamics [j]. Journal of Dalian University of Technology, 2, (1994).

2. Zhong Wanxie. Precise computation for transient analy sis. Computational structural mechanics and applications, 12(1):1-6, (1995).

3. Jiahao Lin, Weiping Shen, and FW Williams. A high precision direct integration scheme for structures subjected to transient dynamic loading. Computers \& structures, 56(1):113-120, (1995).

4. Wang Y X Zhou G. A homogenized high precise direct integration based on taylor series. J Shanghai Jiaotong Univ, 35(1):1916-1919, (2001).

5. Yuexian Wang, Xiaodong Tian, and Gang Zhou. Homogenized high precision direct integration scheme and its applications in engineering. Communications in numerical methods in engineering, 18(6):429-439, (2002).

6. Yuanxian $\mathrm{Gu}$, Biaosong Chen, Hongwu Zhang, and Zhenqun Guan. Precise time-integration method with dimensional expanding for structural dynamic equations. AIAA journal, 39(12):2394-2399, (2001).

7. Gang Zhou Xiaohong Shi. A homogenized high precise direct integration based on legendre series.Chinese Journal of Computational Mechanics, 22(1):335-338, (2001).

8. Gang Zhou Shaohua Fu. A homogenized high precise direct integration based on chebyshev series. Journal of Donghua University, 32(2):46-49, (2006).

9. JH Argyris and DW Scharpf. Finite elements in time and space. Nuclear Engineering and Design, 10(4):456-464, (1969).

10. Wm H Reed and TR Hill. Triangularmesh methodsfor the neutrontransportequation. Los Alamos Report LA-UR-73479, (1973).

11. Bernardo Cockburn and Chi-Wang Shu. The local discontinuous galerkin method for timedependent convection-diffusion systems. SIAM Journal on Numerical Analy sis, 35(6):2440-2463, (1998).

12. M Delfour, W Hager, and F Trochu. Discontinuous galerkin methods for ordinary differential equations. Mathematics of Computation, 36(154):455-473, (1981).

13. Shan Zhao and GW Wei. A unified discontinuous galerkin framework for time integration. Mathematical methods in the applied sciences, 37(7):1042-1071, (2014).

14. C-C Chien and T-YWu. An improved predictor/multicorrector algorithm for a time-discontinuous galerkin finite element method in structural dynamics. Computational Mechanics, 25(5):430-437, (2000).

15. David Cohen, Ernst Hairer, and Ch Lubich. Numerical energy conservation for multi-frequency oscillatory differential equations. BIT Numerical Mathematics, 45(2):287-305, (2005).

16. Xiaomei Liu, Gang Zhou, Yonghong Wang, and Weirong Sun. Rectifying drifts of symplectic algorithm. Beijing University of Aeronautics and Astronautics, 39(1), (2013).

17. Hans Van de Vyver. A symplectic runge-kutta-nystr"om method with minimal phase-lag. Physics Letters A, 367(1):16-24, (2007).

18. Th Monovasilis, Z Kalogiratou, and Tom E Simos. Symplectic partitioned runge-kutta methods with minimal phase-lag. Computer Physics Communications, 181(7):1251-1254, (2010).

19. Qiong Tang, Chuan-miao Chen, and Luo-hua Liu. Energy conservation and symplectic properties of continuous finite element methods for hamiltonian systems. Applied mathematics and computation, 181(2):1357-1368, (2006).

20. Klaus-J"urgen Bathe and Gunwoo Noh. Insight into an 
implicit time integration scheme for structural dynamics. Computers \& Structures, 98:1-6, (2012).

21. Nathan M Newmark. A method of computation for structural dynamics. Journal of the Engineering Mechanics Division, 85(3):67-94, (1959).
22. Edward L Wilson. A computer program for the dynamic stress analy sis of underground structures. Technical report, DTIC Document, (1968). 